\title{
Transcriptional signatures associate with the clinical outcomes of immune checkpoint inhibitor treatment in advanced bladder cancer
}

\author{
Xiaofeng Xu \\ Nanjing University School of Medicine \\ Dian Fu \\ Nanjing University School of Medicine \\ Hai Zhao \\ Nanjing University School of Medicine \\ Jie Huang \\ Nanjing University School of Medicine \\ Zuheng Wang \\ Nanjing University School of Medicine \\ Feng $X u$ \\ Nanjing University School of Medicine \\ Yuhao Chen \\ Nanjing University School of Medicine \\ Jianping Da \\ Nanjing University School of Medicine \\ Tingling Zhang \\ Nanjing University School of Medicine \\ Jingping $\mathrm{Ge}$ \\ Nanjing University School of Medicine \\ Wen Cheng ( $\nabla$ chengwendr@yeah.net ) \\ Nanjing University School of Medicine
}

\section{Research Article}

Keywords: checkpoint blockade, antigen-processing machinery, atezolizumab, PD-1, PD-L1, Urothelial Bladder cancer

Posted Date: October 12th, 2021

DOI: https://doi.org/10.21203/rs.3.rs-889171/v2

License: (c) (i) This work is licensed under a Creative Commons Attribution 4.0 International License. Read Full License 


\section{Abstract \\ Background}

Urothelial bladder cancer (UBC) is one of the most lethal cancers worldwide, the 5-year survival rate remain poor with platinum-based chemotherapy regimens as the standard of cancer treatment protocol. Recent FDA approval of a programmed death ligand-1 (PD-L1) inhibitor, atezolizumab, in advanced UBC patients is changing the therapeutic landscape. Although the response to anti-PD-L1 is correlated to PD-L1 expression and tumor mutation burden, the molecule determinants of responsiveness or non-responsiveness to immune checkpoint inhibitor (ICI) is largely unknown.

\section{Result}

A published immunotherapy cohort with whole exome sequencing, RNAseq and clinic outcome data for 29 metastatic urothelial cancer patients was used, paralleled with The Cancer Genome Altas Bladder Cancer cohort for validation. Genomic mutational profiling, mutational signature, a panel genes in antigen presentation and interferon signaling in bladder cancer were delineated with little correlation with durable clinic benefit (DCB) or non-DCB of PDL1 inhibitor treatment. Whereas, characterized immune-responsive or resistant associated genes showed differentially expressed between $D C B$ group and non-DCB group. Further more, transcriptional signature and transcriptional regulators between $\mathrm{DCB}$ and non-DCB were identified from transcripome data.

\section{Conclusion}

Our exploratory analyses provided multidimension view of complexity of determinants of immune-responsiveness and suggest the influences of transcriptional reprogram in immune checkpoint blockage therapy.

\section{Introduction}

Urothelial bladder cancer (UBC) is the most common cancer from urinary tract worldwide, causing 150,000 deaths per year, and it is characterized with high rate of relapse, metastasis, and mortality [1]. Median survival for patients with recurrent or metastatic bladder cancer remains 14-15 months with cisplatin-based chemotherapy, and there is no widely recognized second-line therapy[2, 3]. Immunotherapy has played an essential role in UBC with the use of Baccille Calmette Guerin (BCG) in the treatment of non-muscle invasive bladder cancer [4]. Recent approval of a PDL1 inhibitor, atezolizumab, in patients with locally advanced or metastatic urothelial carcinoma who have progressed following treatment with platinum-based chemotherapy [5] or who is ineligible to cisplatin[6] represents a significant milestone in treating urothelial cancer in the past 30 years. In these two clinical trials, patients were selected based on PD-L1 expression on tumor cells or immune cells, the over call objective response rate is about $15 \% \sim 23 \%$, tumor mutation burden is associated to the response $[5,6]$. The determinants of response and resistance to immune checkpoint inhibitors (ICls) is a key to improving outcomes and developing new treatment strategies. Genomic and transcriptomic characteristics have been demonstrated to be correlated with response to anti-PD-1, CTLA-4 treatment in metastatic melanoma [7-9], non-small cell lung cancer [10] or colorectal cancer [11, 12]. As of metastatic urothelial cancer, high neoantigen or tumor mutational burden, CD8 + T-effector cell phenotype are associated with response to treatment, a signature of transforming growth factor $\beta$ (TGF $\beta$ ) signalling in fibroblasts is associated with non-response [13], more importantly, the balance of adaptive immunity and protumorigenic inflammation within 
tumor microenvironments was reported to associate with PD-1/PD-L1 inhibitor resistance [14]. Therefore, it is crucial to continuously identify biomarker(s) to stratify or predict responders to immune checkpoint blockade for better clinical outcome using different cohorts.

In this study, we analyzed whole exome sequence and RNA-seq data of urothelial bladder cancer patients who received PD-L1 inhibitor treatment and the clinical outcomes were categorized into patients with durable clinic benefit (DCB) or without durable clinic benefit (non-DCBs). The Cancer Genome Altas (TCGA) was also used for crossvalidation. We provided genomic and transcriptomic foundation to understand mechanisms of bladder cancer patients not only whether respond to anti-PD-L1 treatment, but also the long-lasting clinic benefit.

\section{Material And Methods}

1. Somatic mutations shared by bladder cancer patients in COSMIC database.

The frequency of the specific point mutation in each gene mutation was estimated as following: (1) genetic mutation data within the top 500 sample numbers of bladder cancer (transition cell carcinoma) were downloaded from COSMIC (GRCh38 v94). COSMIC > > Cancer Browser > > Urinary tract from Tissue selection > > Bladder from Subtissue $>>$ Carcinoma from Histology selection $>>$ Transitional cell carcinoma from subHistology selection.

2. Bladder urothelial carcinoma (BLCA) whole exome raw mutation annotation file $(n=395)$ was downloaded from firehose broad institute (firebrowse.org), gene expression RNAseq (polyA + IlluminaHiSeq, n= 426, level 3) and phenotype $(n=436)$ was queried from UCSC Xena browser (https://xenabrowser.net/datapages/? cohort=TCGA\%20Bladder\%20Cancer\%20(BLCA)\&removeHub=https\%3A\%2F\%2Fxena.treehouse.gi.ucsc.edu\%3A443). The data was processed in R statistic program with maftools, GenVisR packages.

3. Locally advanced or metastatic bladder cancer patients $(n=29)$ from Memorial Sloan Kettering Cancer Center were treated with atezolizumab were on protocol NCT02108652 [5]. Their whole exome sequence, bulk RNAseq and clinical information were downloaded from http://doi.org/10.5281/zenodo.546110 and

https://github.com/hammerlab/multi-omic-urothelialanti-pdl1. Mechanistic-driven analyses were performed on these data.

4. Differentially expressed genes (DEGs). Gene expression matrix from RNAseq data of 26 advanced bladder cancer cases receiving PD-L1 inhibitor treatment were analyzed using EdgeR and limma R package with default parameters. The absolute $\log 2 \mathrm{FC}>=1.5$ and false discovery rate $<=0.05$ was used as cut-off, thirty-three DEGs were selected for heatmap plot using ComplexHeatmap package.

5. Dysfunctional regulations of gene expression program in PD-L1 inhibitor treated urothelial bladder cancer cohort. Gene expression from DCB $(n=9)$ and non-DCB groups $(n=17)$ of advanced bladder cancer cases receiving PD-L1 inhibitor treatment were analyzed using DysRegSig algorithm in $\mathrm{R}$ packages.

\section{Results}

\section{Mutational burden and signature in bladder cancer}

Whole exome sequencing for 391 bladder cancer samples were obtained from The Cancer Genome Atlas (TCGA), non-synonymouse mutation was retained for analysis. The total number of non-synonymous mutations in each patient is heterogeneous with median number of 173.5 (Fig. 1A). Somatic point mutations have two types of DNA substitution: 
transitions ( $A \leftrightarrow G$ and $C \leftrightarrow T$ ) and transversions $(A \leftrightarrow C, G \leftrightarrow T, A \leftrightarrow T, C \leftrightarrow G)$. Transition mutation is higher than transversion mutation, and $C>T$ transition and $C>G$ transversion are higher than other type of mutations in average and in each individual case (Fig. 1B, C). We then analyzed the mutational signature against known mutational signatures[15], three predominant signatures were defined that highly correlated with APOBEC signature (signature 2 , 13), aging signature (signature 1) and ultra-hypermutators with error-prone polymerase POLE somatic mutation (Signature 10) (Fig. 1D). These broad mutation processes in bladder cancers corresponded with the hyper mutation rate in bladder cancer genome and correlated to PD-L1 high/CD8A high subpopulation [16]. Recently, Snyder et al [17]systematically analyzed multi-omic data from subset patients of immune checkpoint inhibitor, atezolizumab, clinical trial cohort. We take advantage of whole exon sequencing data from this subset cohort, compared the genomic difference between patients with durable clinic benefit (DCB, response lasting more than 6 months), and patients without durable clinic benefit (non-DCB). We observed that higher average mutation variants in DCB group than non-DCB group (unpaired two sample t-test, $\mathrm{p}<0.05$ ) (Supplementary Fig. 1), this is consistent with higher mutation burden in responders relative to non-responders [13]. Interestingly, the distribution of mutation types ( $T>G$, $T>A, T>C, C>T, C>G, C>A$ ) in DCB and non-DCB groups are similar. We obtained top 20 frequent mutated cancer genes in bladder cancer from COSMIC (supplementary Fig. 2), we did not observe COSMIC cancer mutated genes enriched in either group, nor significant differential mutated genes or the mutated gene involved pathways between two groups (Supplementary Fig. 3).

Interferon-receptor signaling and antigen presentation pathways defects in bladder cancer. Immune checkpoint blockade therapy on melanoma, lung cancer and colorectal cancer provided underlying mechanisms of responding, resistance to PD-1 or CTLA-4 inhibitors. From genomic level, JAK1, JAK2 and B2M have found associated with acquired resistance to anti-PD-1 in melanoma [9]. Positive selection for HLA and antigen- processing machinery mutations in tumors with TILs may have implications for potential immunotherapy resistance [12]. Castro et al reported that B2M mutation (occurring relatively early event in tumors) and HLA mutations were highly enriched in patients with microsatellite instability. In addition, these mutations had higher levels of immune infiltration by natural killer and CD8 + T cells and higher levels of cytotoxicity[18]. From TCGA bladder cancer patients $(n=396)$ with whole exome sequence data, we found 66 ( 17\%) patients harbored mutations associated with either MHC binding molecules (HLA gene A, B, C), antigen-processing machinery (APM) pathway and interferon receptor signaling. Interestingly, these mutations exhibit mutually exclusive mutation pattern, indicating the roles of these genes in immune response regulation (Fig. 2A). We wondered that how these gene mutations in the ICl-treated cohort. We found that JAK1, PDIA3, CALR, TAP1, TAPBP have higher mutation frequency in non-DCB group than in DCB group. Other gene mutation rate is higher in DCB group than in non-DCB group although there is no significant statistical difference due to a small sample size (Fig. 2B). Taken together, genetic alterations seem to exhibit limited correlated with durable clinical benefit from ICI treatment.

\section{Putative immune checkpoint sensitivity genes expression in bladder cancer}

From transcriptional level, a subset of genes such as granzyme A (GZMA), perforin (PRF1, CD8 T cell cytolytic score), PDCD1LG2 (PD-L2), and CTLA4 were expressed higher in the pretreatment melanoma tumors of patients who exhibited benefit from CTLA-4 antibodies [19]. Other surface receptors PDCD1 (PD-1), LAG3, HAVCR2 (Tim-3), CD160 and CD244 as well as transcription factors EOMES, PRDM1 (Blimp-1), and TBX21 (T-BET) were identified from T cell exhaustion [20]. We were wondering how these gene expressions changed in ICl-treated bladder cancer patients. From bulk RNAseq data, we observed that CD8A, CD8B, PDCD1(PD-1), PRF1, GZMA, EOMES, and TBX21 highly expressed in DCB group than in non-DCB group ( $<<0.2$, unpaired two sample t-test) (Figure $3 A$ ). Hierarchical cluster of the correlation of these genes showed different patterns between two groups (Supplementary Figure 4). We then 
estimated the relative abundance of different cell types in the tumor microenvironment (TME) using bulk tumor RNAseq data with advanced concensusTME algorithm [21]. In ICl-treated cohort, among 19 different cell types within TME, the abundances of mast cells and fibroblast cells are marginally higher in non-DCB than in DCB group ( $p=0.082$ and $p=0.16$, respectively. Supplementary Figure 5 ). Tumor-infiltrating mast cells have recently reported to associate with resistance to anti-PD-1 therapy in humanized melanoma mouse model [22]. Tumor-associated fibroblasts could activate transforming growth factor $\beta$ signaling resulting in restricting T-cell infiltration and restrain anti-tumor immunity in urothelial cancer [13].

Because the numbers of tumor-infiltrating CD8+ T cells (TILs) increase overall survival and immune checkpoint inhibitor efficacy. From RNA-seq data, the expression of CD8A (one component of the CD8 dimer) has been as a surrogate for TIL levels [23]. Within 390 bladder cancer patients with clinical information and RNA-seq data, bladder cancer with high CD8A expression has significantly higher overall survival time than that with lower CD8A expression $(p<0.05)$. (Figure $3 B)$. We observed that CD8A and CD8+ T cell showed the high expression trend in DCB group compared to non-DCB group. These positive findings support the TIL cells and antigen recognition by T cells in DCB group.

\section{Differentially expressed genes and transcription regulators between DCB and non-DCB.}

The $29 \mathrm{ICl}$-treated cohort has been exploratory analyzed by Snyder et al, mainly focusing on genomic alteration and T cell receptor functions [17]. We took advantage of RNAseq from pre-treated samples to examinate the differentially expressed genes (DEGs) and master regulators between patients with DCB and non-DCB. Figure 4A showed 33 DEGs (absolute log2FC >1.5 and false discovery rate $<0.1)$. Among them, 9 genes $(27.8 \%)$ are down-regulated in DCB and 24 genes $(72.2 \%)$ are up-regulated in DCB. These genes involved in multiple GO biological processes such as oxidative stress, metabolism and cell-cell adhesion (Figure 4B). Interestingly, there are several non-coding transcripts in DEGs, their biological functions remain elusive.

In order to deeply understand the transcriptional regulation, we used DysRegSig algorithm, a machine learning-based gene dysregulation analysis [24], to explore the gene expression dysregulation between DCB and non-DCB patients, there are 184 significantly dysregulated transcription factor (TF) - targeted genes pairs (Supplementary Table 1). The dysregulated target genes are predominately enriched in immune-related signallings such as TNFs, STAT5-IL2-T cell activation, TNFR2 non-canonical NF-KB pathway, IL-18 signaling and cytokine-cytokine receptor interaction (Supplementary Table 2). Further gene regulatory network analysis revealed 10 top-ranked master transcriptional regulators (Supplementary Table 3). One of them is PATZ1-WNK4 with regulatory intensity 3.107, which is highly correlated to DCB phenotype (Figure 5). Transcription factor PATZ1 negatively regulates the development of FOXP3 regulatory T cells [25], this may contribute to patients with good clinic benefit when receiving ICI treatment.

\section{Discussion}

Remarkable clinical efficacy, durable response and low toxicity of immune checkpoint blockade treatment have been observed in various malignancies including UBC $[5,6,26]$. anti-PD-L1 for advanced bladder cancer could reach $43.3 \%$ response rate in early trial small cohort[26], and $>10 \%$ in later large cohort $[5,6]$. However, a large proportion of patients failed to respond to checkpoint inhibitors, therefore, it is crucial to identify biomarker(s) to stratify or predict responders to achieve better clinical outcome.

The molecular determinants of responsiveness to PD-1/PD-L1 and CTLA-4 inhibitors appear to be heterogeneous and complex. We take advantage of PD-L1 inhibitor treated bladder cancer cohort and TCGA bladder cancer data to 
analyze the molecular determinants of immuno-sensitivity or resistance in bladder cancer. First, urothelial bladder cancer is a genomic disorder with high mutation load. Three dominant mutation signatures have been identified such as APOBEC, POLE signature and aging signature. The 29 PD-L1 inhibitor (Atezolizumab) treated patients with advanced bladder cancer did not have recurrent gene mutations correlated to DCB group or non-DCB group from WES, only positive genomic feature association to DCB is higher tumor mutation burden as reported previously [13]. Second, we found $17 \%$ bladder cancer patients from TCGA-BLCA cohort harboring mutations involving MHC molecules, antigen processing machinery or interferon-receptor signaling pathway. These mutations exhibited mutually exclusive pattern. In the ICl-treated cohort, we observed that JAK1, TAPBP mutation frequency is relative higher in non-DCB group than CDB group. Third, a panel of nineteen immuno-responsive genes in other types of cancers differentially expressed in DCB group and non-DCB group of bladder cancer patients who received ICI treatment, such as CD8A, CD8B, GZMA (granzyme A), transcription factor EOMES, TBX21 marginally higher in DCB relative to non-DCB, these gene expressions indicate functional activities of immunity. More over, patients with high CD8A expression demonstrated favorable clinical outcome. Finally, we identified differentially expressed genes between DCB group and non-DCB group. These transcriptional signature is able to discriminate ICl-treated clinic outcomes. Ten significant transcriptional regulators were further characterized from the RNAseq, which reflects transcriptional reprogramming tumor cells and or tumor microenvironment that influence the consequence of immune checkpoint blockade therapy.

transcriptional signatures are potential predictors for responding to antagonists of PD-1. For instance, up-regulation of mesenchymal transition genes ( $A X L, R O R 2, W N T 5 A, L O X L 2, T W I S T 2, T A G L N, F A P)$, immunosuppressive genes (IL10,VEGFA,VEGFC) and monocyte and macrophage chemotactic genes (CCL2, CCL7, CCL8, CCL 13) preferentially in non-responding tumors [8], indicating patients with these expression signature most likely do not respond to antiPD-1 treatment. These findings highlight the complexity interplay between cancer cells and the immune system which will need further elucidation in urothelial bladder cancer.

The limitation of this analysis is that the TCGA-BLCA cohort did not have PD-1 or PD-L1 inhibitor treatment information, and the ICl-treated metastatic bladder cancer cohort contains small samples, particularly patients with durable clinic benefit group. Most the difference between DCB and non-DCB group are marginal. The findings remain to be confirmed in a large data in the future.

\section{Declarations}

\section{Consent for publication: Not applicable}

Availability of data and materials: The datasets analyzed during the current study are public available from weblinks: Somatic mutations shared by bladder cancer patients in COSMIC database (https://cancer.sanger.ac.uk/cosmic/browse/tissue). TCGA-BLCA whole exome mutation annotation file was downloaded from firehose broad institute (http://firebrowse.org/?cohort=BLCA). TCGA-BLCA RNAseq data was downloaded from UCSC Xena browser (https://xenabrowser.net/datapages/? cohort=TCGA\%20Bladder\%20Cancer\%20(BLCA)\&removeHub=https\%3A\%2F\%2Fxena.treehouse.gi.ucsc.edu\%3A443). whole exome sequence, bulk RNAseq and clinical information derived from 29 locally advanced or metastatic bladder cancer patients (Memorial Sloan Kettering Cancer Center) were downloaded from http://doi.org/10.5281/zenodo.546110 and https://github.com/hammerlab/multi-omic-urothelialanti-pdl1, respectively. 
Funding: Natural Science Foundation of China through grant 81572526 and 81972841, the fifth phase of "333 Highlevel Talent Cultivation Project” in Jiangsu Province (BRA2018097), Project of Jiangsu Provincial Health Committee (Z2018020)

Conflict of Interests: None

Authors' contributions: Conceived by WC, investigated by X.F. Xu, DF, HZ, JH, data collection and analysis with ZW, FX Y.H.C. J.P.D, T.L.Z., reviewed and edited by J.P.G.

Acknowledgement: This research was supported by Natural Science Foundation of China through grant 81572526 and 81972841, the fifth phase of "333 High-level Talent Cultivation Project" in Jiangsu Province (BRA2018097), Project of Jiangsu Provincial Health Committee (Z2018020).

\section{References}

1. Torre LA, Bray F, Siegel RL, Ferlay J, Lortet-Tieulent J, Jemal A: Global cancer statistics, 2012. CA Cancer J Clin 2015, 65:87-108.

2. von der Maase H, Sengelov L, Roberts JT, Ricci S, Dogliotti L, Oliver T, Moore MJ, Zimmermann A, Arning M: Long-term survival results of a randomized trial comparing gemcitabine plus cisplatin, with methotrexate, vinblastine, doxorubicin, plus cisplatin in patients with bladder cancer. J Clin Oncol 2005, 23:4602-4608.

3. Sonpavde G, Sternberg CN, Rosenberg JE, Hahn NM, Galsky MD, Vogelzang NJ: Second-line systemic therapy and emerging drugs for metastatic transitional-cell carcinoma of the urothelium. Lancet Oncol 2010, 11:861870.

4. Lenis AT, Chamie K: Bladder cancer in 2014: From the genomic frontier to immunotherapeutics. Nat Rev Urol $2015,12: 74-76$.

5. Rosenberg JE, Hoffman-Censits J, Powles T, van der Heijden MS, Balar AV, Necchi A, Dawson N, O'Donnell PH, Balmanoukian A, Loriot $Y$, et al: Atezolizumab in patients with locally advanced and metastatic urothelial carcinoma who have progressed following treatment with platinum-based chemotherapy: a single-arm, multicentre, phase 2 trial. Lancet 2016, 387:1909-1920.

6. Balar AV, Galsky MD, Rosenberg JE, Powles T, Petrylak DP, Bellmunt J, Loriot Y, Necchi A, Hoffman-Censits J, Perez-Gracia JL, et al: Atezolizumab as first-line treatment in cisplatin-ineligible patients with locally advanced and metastatic urothelial carcinoma: a single-arm, multicentre, phase 2 trial. Lancet 2017, 389:67-76.

7. Snyder A, Makarov V, Merghoub T, Yuan J, Zaretsky JM, Desrichard A, Walsh LA, Postow MA, Wong P, Ho TS, et al: Genetic basis for clinical response to CTLA-4 blockade in melanoma. N Engl J Med 2014, 371:2189-2199.

8. Hugo W, Zaretsky JM, Sun L, Song C, Moreno BH, Hu-Lieskovan S, Berent-Maoz B, Pang J, Chmielowski B, Cherry G, et al: Genomic and Transcriptomic Features of Response to Anti-PD-1 Therapy in Metastatic Melanoma. Cell 2016, 165:35-44.

9. Zaretsky JM, Garcia-Diaz A, Shin DS, Escuin-Ordinas H, Hugo W, Hu-Lieskovan S, Torrejon DY, Abril-Rodriguez G, Sandoval S, Barthly L, et al: Mutations Associated with Acquired Resistance to PD-1 Blockade in Melanoma. N Engl J Med 2016, 375:819-829.

10. Rizvi NA, Hellmann MD, Snyder A, Kvistborg P, Makarov V, Havel JJ, Lee W, Yuan J, Wong P, Ho TS, et al: Cancer immunology. Mutational landscape determines sensitivity to PD-1 blockade in non-small cell lung cancer. Science 2015, 348:124-128. 
11. Le DT, Uram JN, Wang H, Bartlett BR, Kemberling H, Eyring AD, Skora AD, Luber BS, Azad NS, Laheru D, et al: PD-1 Blockade in Tumors with Mismatch-Repair Deficiency. N Engl J Med 2015, 372:2509-2520.

12. Giannakis M, Mu XJ, Shukla SA, Qian ZR, Cohen O, Nishihara R, Bahl S, Cao Y, Amin-Mansour A, Yamauchi M, et al: Genomic Correlates of Immune-Cell Infiltrates in Colorectal Carcinoma. Cell Rep 2016, 15:857-865.

13. Mariathasan S, Turley SJ, Nickles D, Castiglioni A, Yuen K, Wang Y, Kadel EE, III, Koeppen H, Astarita JL, Cubas R, et al: TGFbeta attenuates tumour response to PD-L1 blockade by contributing to exclusion of T cells. Nature 2018, 554:544-548.

14. Wang L, Sfakianos JP, Beaumont KG, Akturk G, Horowitz A, Sebra RP, Farkas AM, Gnjatic S, Hake A, Izadmehr S, et al: Myeloid Cell-associated Resistance to PD-1/PD-L1 Blockade in Urothelial Cancer Revealed Through Bulk and Single-cell RNA Sequencing. Clin Cancer Res 2021.

15. Alexandrov LB, Nik-Zainal S, Wedge DC, Aparicio SA, Behjati S, Biankin AV, Bignell GR, Bolli N, Borg A, BorresenDale AL, et al: Signatures of mutational processes in human cancer. Nature 2013, 500:415-421.

16. Chen S, Zhang N, Shao J, Wang T, Wang X: Multi-omics Perspective on the Tumor Microenvironment based on PD-L1 and CD8 T-Cell Infiltration in Urothelial Cancer. J Cancer 2019, 10:697-707.

17. Snyder A, Nathanson T, Funt SA, Ahuja A, Buros Novik J, Hellmann MD, Chang E, Aksoy BA, Al-Ahmadie H, Yusko $\mathrm{E}$, et al: Contribution of systemic and somatic factors to clinical response and resistance to PD-L1 blockade in urothelial cancer: An exploratory multi-omic analysis. PLoS Med 2017, 14:e1002309.

18. Castro A, Ozturk K, Pyke RM, Xian S, Zanetti M, Carter H: Elevated neoantigen levels in tumors with somatic mutations in the HLA-A, HLA-B, HLA-C and B2M genes. BMC Med Genomics 2019, 12:107.

19. Van Allen EM, Miao D, Schilling B, Shukla SA, Blank C, Zimmer L, Sucker A, Hillen U, Geukes Foppen MH, Goldinger SM, et al: Genomic correlates of response to CTLA-4 blockade in metastatic melanoma. Science 2015, 350:207-211.

20. Wherry EJ: T cell exhaustion. Nat Immunol 2011, 12:492-499.

21. Jimenez-Sanchez A, Cast O, Miller ML: Comprehensive Benchmarking and Integration of Tumor Microenvironment Cell Estimation Methods. Cancer Res 2019, 79:6238-6246.

22. Somasundaram R, Connelly T, Choi R, Choi H, Samarkina A, Li L, Gregorio E, Chen Y, Thakur R, Abdel-Mohsen M, et al: Tumor-infiltrating mast cells are associated with resistance to anti-PD-1 therapy. Nat Commun 2021, 12:346.

23. Brown SD, Warren RL, Gibb EA, Martin SD, Spinelli JJ, Nelson BH, Holt RA: Neo-antigens predicted by tumor genome meta-analysis correlate with increased patient survival. Genome Res 2014, 24:743-750.

24. Li Q, Dai W, Liu J, Sang Q, Li YX, Li YY: DysRegSig: an R package for identifying gene dysregulations and building mechanistic signatures in cancer. Bioinformatics 2021, 37:429-430.

25. Andersen L, Gulich AF, Alteneder M, Preglej T, Orola MJ, Dhele N, Stolz V, Schebesta A, Hamminger P, Hladik A, et al: The Transcription Factor MAZR/PATZ1 Regulates the Development of FOXP3(+) Regulatory T Cells. Cell Rep 2019, 29:4447-4459 e4446.

26. Powles T, Eder JP, Fine GD, Braiteh FS, Loriot Y, Cruz C, Bellmunt J, Burris HA, Petrylak DP, Teng SL, et al: MPDL3280A (anti-PD-L1) treatment leads to clinical activity in metastatic bladder cancer. Nature 2014, 515:558-562.

\section{Figures}


A

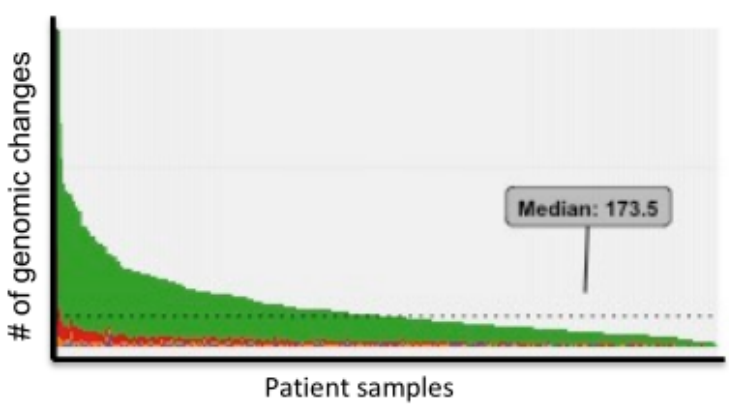

C

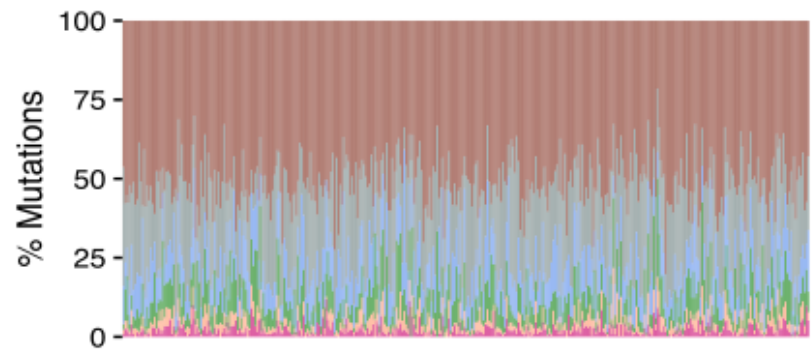

Samples

$\mathrm{C}>\mathrm{T} \quad \mathrm{C}>\mathrm{A} \quad \mathrm{T}>\mathrm{A}$

$C>G \quad T>C \quad T>G$
B
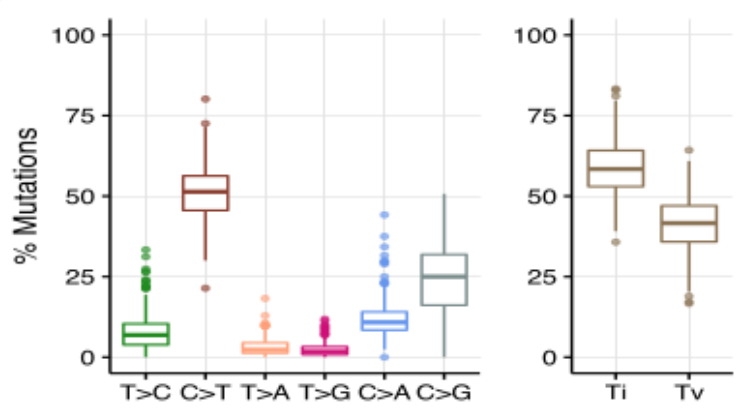

D
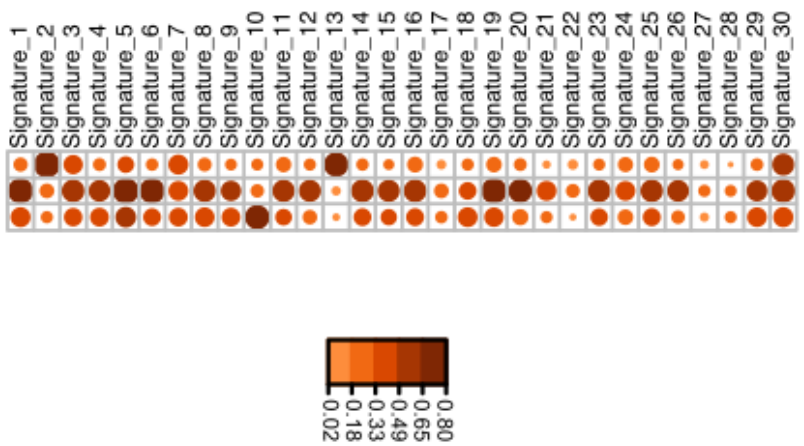

Figure 1

\section{Figure 1}

Mutational landscape of urothelial bladder cancer. Whole exom sequence data for TCGA-BLCA were used to investigate the tumor mutational burden $(A)$, mutation type distribution $(B, C)$ and somatic mutational signatures (D). 
A

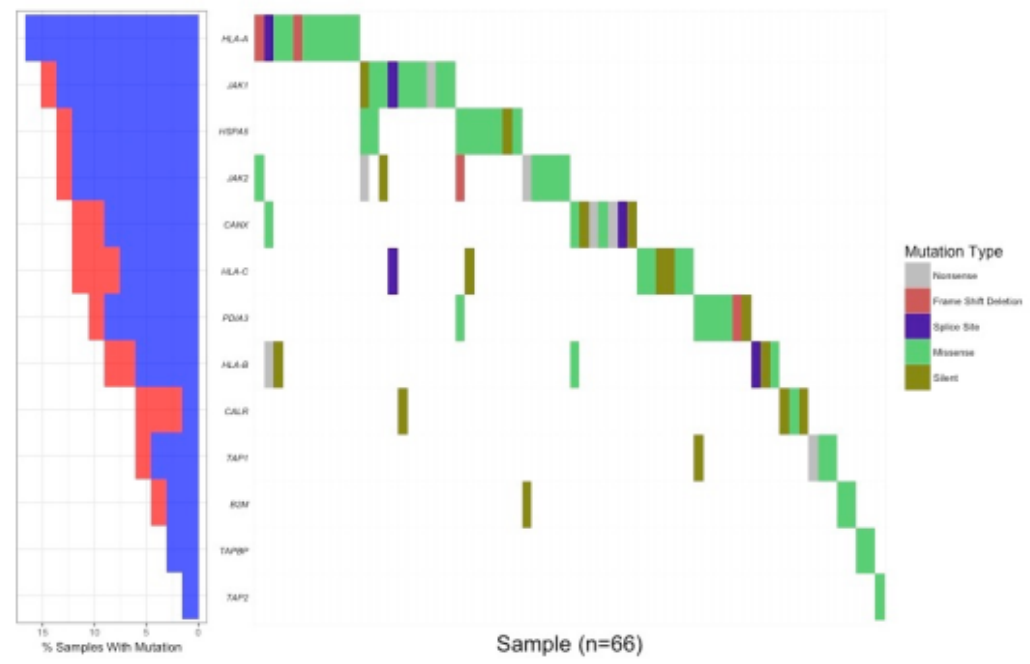

B

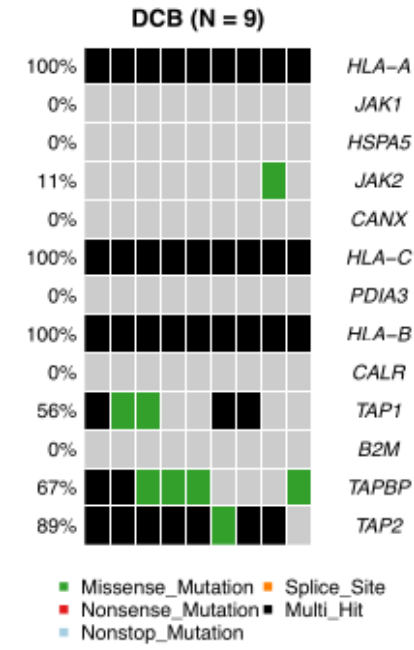

Non-DCB $(\mathrm{N}=17)$

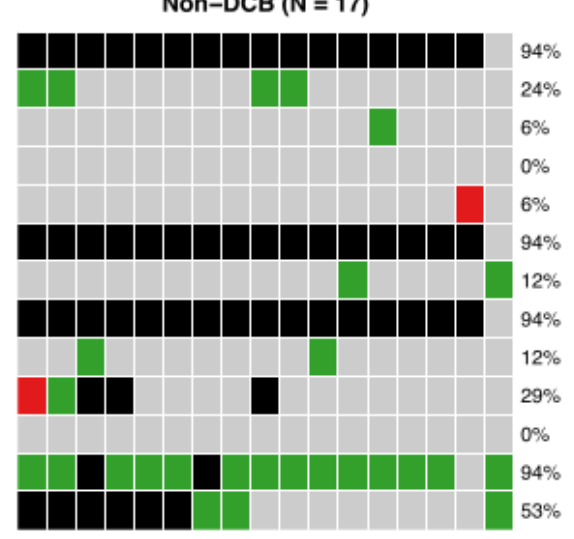

Figure 2

\section{Figure 2}

Mutational profiling of genes in antigen-processing machinery and interferon-receptor signaling pathway in urothelial bladder cancer. (A) The oncoprint of 19 genes participating antigen-processing machinery and interferon-receptor signaling pathway from TCGA-BLCA was presented. (B) The immune checkpoint inhibitor-treated bladder cancer cohort was grouped as durable clinical benefit (DCB) and non durable clinical benefit (non-DCB). Target gene mutational profiling was demonstrated in each group. The color codes represent different type of mutations. 
A

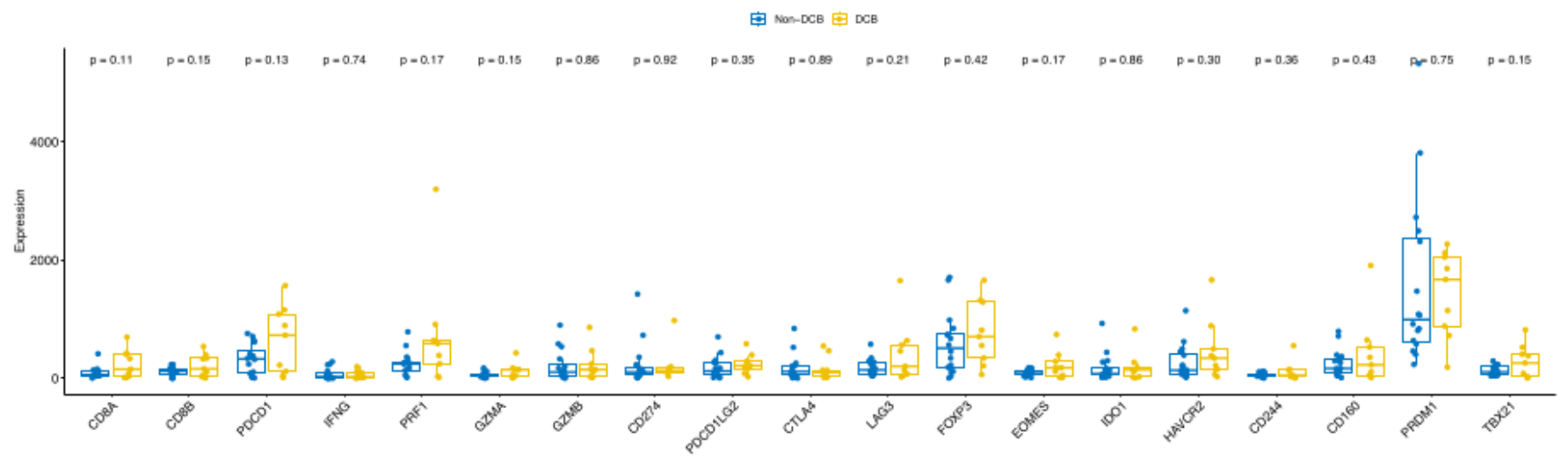

B

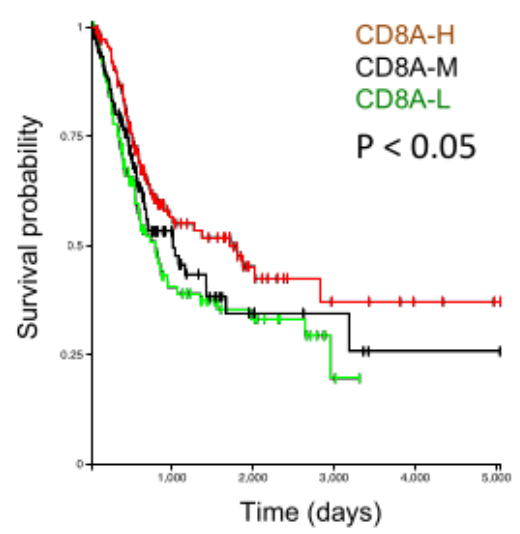

Figure 3

\section{Figure 3}

Immune-related gene expressions and clinic association in urothelial bladder cancer. (A) Selected immune checkpoint inhibitor treatment related gene panel of expression in durable clinic benefit (DCB) group and non-DCB group. (B) Kapan Miere survival plot showed the level of CD8A expression (proxy of tumor infiltrating lymphocytes, TIL) and overall survival time in TCGA-BLCA cohort. 
A

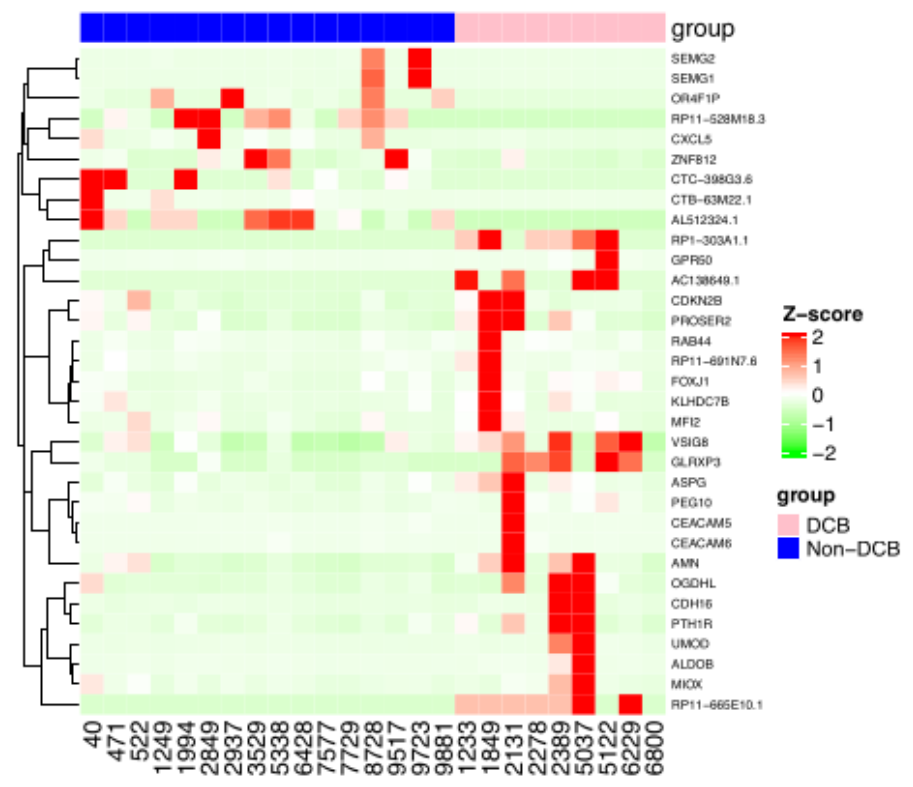

B

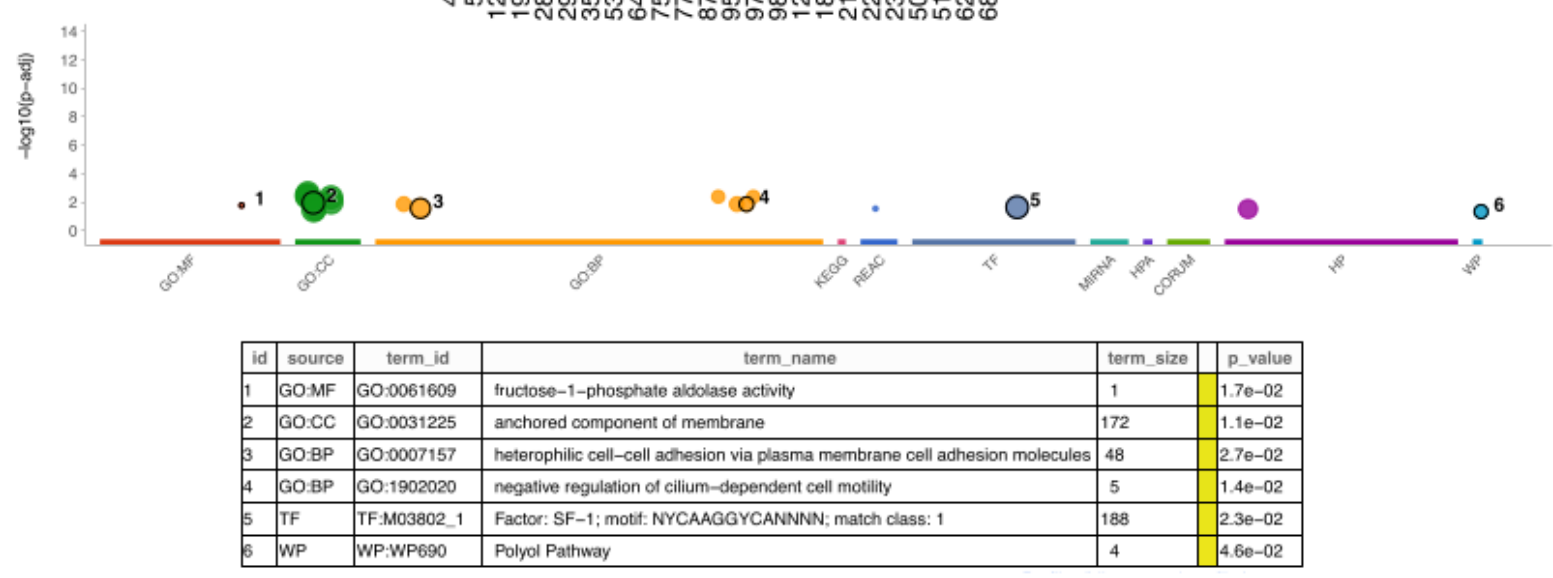

Figure 4

Figure 4

Differentially expressed genes between bladder cancer patients presenting durable clinic benefit (DCB) and non-DCB after PD-L1 inhibitor treatment. (A) Bulk RNAseq was derived from pretreatment tumor samples, the differentially expressed genes were identified based on $|\log 2(\mathrm{FC})|>=1.5$, and $\mathrm{FDR}<0.05$, and visualized in heatmap. (B) The GO terms of Differentially expression genes were analyzed with goprofiler with multiple pathway databases. The selected representative GO terms were listed in the table below the plot. p-value for the enrichment $<0.05$. 


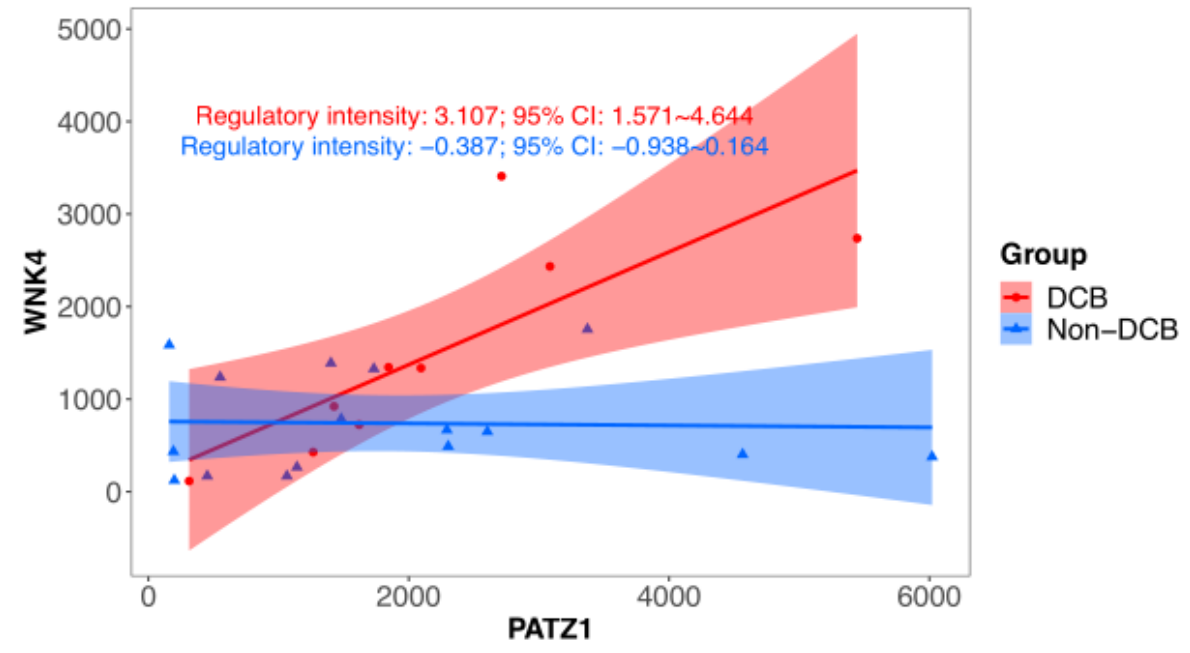

Figure 5

\section{Figure 5}

Representative dysregulator and target in immune PD-L1 inhibitor treated bladder cancer. RNAseq derived from advanced bladder cancer patients with durable clinic benefit (DCB) group and non-DCB group was subjected to systematically identification of dysregulation events associated with treatment benefit using DysRegSig algorithm. The top one ranked transcription factor (PATZ1) and target (MNK4) was highly correlated in DCB group compared to non-DCB group. The regulatory intensity and $95 \%$ confidence interval was shown in each group.

\section{Supplementary Files}

This is a list of supplementary files associated with this preprint. Click to download.

- CWpaper2021SupplFigures.pdf

- SupplementaryTable1.txt

- SupplementaryTable2.txt

- SupplementaryTable3.txt 